Fumihiro Akiyama - Toshihiro Tanaka - Ryo Yamada

Yozo Ohnishi · Tatsuhiko Tsunoda - Shiro Maeda

Takashi Takei · Wataru Obara $\cdot$ Kyoko Ito

Kazuho Honda • Keiko Uchida • Ken Tsuchiya

Kosaku Nitta • Wako Yumura • Hiroshi Nihei

Takashi Ujiie • Yutaka Nagane • Satoru Miyano

Yasushi Suzuki · Tomoaki Fujioka • Ichiei Narita

Fumitake Gejyo $\cdot$ Yusuke Nakamura

\title{
Single-nucleotide polymorphisms in the class II region of the major histocompatibility complex in Japanese patients with immunoglobulin A nephropathy
}

\author{
Received: May 28, 2002 / Accepted: July 4, 2002
}

Abstract Immunoglobulin A nephropathy $(\operatorname{Ig} A N)$ is a form of chronic glomerulonephritis of unknown etiology and pathogenesis. Immunogenetic studies have not conclusively indicated that human leukocyte antigen (HLA) is

F. Akiyama $\cdot$ W. Obara $\cdot$ S. Miyano $\cdot$ Y. Nakamura

Human Genome Center, The Institute of Medical Science,

University of Tokyo, Tokyo, Japan

F. Akiyama · I. Narita · F. Gejyo

Division of Clinical Nephrology and Rheumatology, Niigata

University Graduate School of Medical and Dental Sciences, Niigata, Japan

T. Tanaka $\cdot$ Y. Ohnishi

Laboratory for Cardiovascular Diseases, SNP Research Center, The Institute of Physical and Chemical Research (RIKEN), Tokyo, Japan

R. Yamada

Laboratory for Rheumatic Diseases, SNP Research Center, The

Institute of Physical and Chemical Research (RIKEN), Tokyo, Japan

T. Tsunoda

Laboratory for Medical Informatics, SNP Research Center, The

Institute of Physical and Chemical Research (RIKEN), Tokyo, Japan

S. Maeda

Laboratory for Diabetic Nephropathy, SNP Research Center, The

Institute of Physical and Chemical Research (RIKEN), Tokyo, Japan

T. Takei $\cdot$ K. Ito $\cdot$ K. Honda $\cdot$ K. Uchida $\cdot$ K. Tsuchiya $\cdot$ K. Nitta $\cdot$

W. Yumura $\cdot \mathrm{H}$. Nihei

Department of Medicine, Kidney Center, Tokyo Women's Medical

University, Tokyo, Japan

T. Ujiie

Department of Urology, Iwate Prefectural Ofunato Hospital, Iwate, Japan

Y. Nagane

Department of Urology, Sanai Hospital, Iwate, Japan

Y. Suzuki · T. Fujioka

Department of Urology, Iwate Medical University, Iwate, Japan

Y. Nakamura $(\bowtie)$

Laboratory of Molecular Medicine, Human Genome Center,

Institute of Medical Science, University of Tokyo, 4-6-1

Shirokanedai, Minato-ku, Tokyo 108-8639, Japan

Tel. +81-3-5449-5372; Fax +81-3-5449-5433

e-mail: yusuke@ims.u-tokyo.ac.jp involved. As a first step in investigating a possible relationship between HLA class II genes and IgAN, we analyzed the extent of linkage disequilibrium (LD) in this region of chromosome 6 p21.3 in a Japanese test population and found extended LD blocks within the class II locus. We designed a case-control association study of singlenucleotide polymorphisms (SNPs) in each of those LD blocks, and determined that SNPs located in the $H L A$ $D R A$ gene were significantly associated with an increased risk of $\operatorname{IgAN}(P=0.000001$, odds ratio $=1.91[95 \%$ confidence interval 1.46-2.49]); SNPs in other LD blocks were not. Our data imply that some haplotype of the $H L A$ $D R A$ locus has an important role in the development of IgAN in Japanese patients.

Key words Single-nucleotide polymorphism $\cdot \operatorname{IgA}$ nephropathy $\cdot$ Linkage disequilibrium $\cdot$ HLA class II $\cdot H L A-D R A$

\section{Introduction}

Immunoglobulin A nephropathy (IgAN [MIM161950]), a disease characterized by predominant IgA deposits in glomerular mesangial areas, is the most common type of glomerulonephritis (GN); its prevalence may be as high as $50 \%$ of all cases of GN in Asia, especially among the Japanese. Long-term follow-up studies of biopsy-proven cases of $\operatorname{IgAN}$ have revealed that $20 \%-30 \%$ of patients progress to end-stage renal disease within 20 years of GN onset (Galla 1995; Floege and Feehally 2000).

The pathogenesis of $\operatorname{IgAN}$ is unknown, but accumulated data suggest that some genetic factors are involved in disease susceptibility (Galla 2001). The prevalence of IgAN seems to reflect demographic and ethnic characteristics of the populations studied; moreover, several cases of familial IgAN (Julian et al. 1985; Scolari et al. 1999) and higher risk of identical twins to IgAN (Tolkoff-Rubin et al. 1978; Sabatier et al. 1979) have been reported. Investigators have 
also observed an increased frequency of specific human leukocyte antigens (HLAs) in some patient populations (Hsu et al. 2000).

Although numerous studies have focused on HLAs encoded by the human major histocompatibility complex (MHC) locus with respect to possible linkage with susceptibility to $\operatorname{IgAN}$, no consistent results have emerged (Hsu et al. 2000). Lately, however, genes encoding HLAs have come to be considered useful markers for identifying disease-susceptibility loci, rather than causing diseases themselves (Moore 1993; Schena 1995). This concept implies that loci linked to HLA genes could be associated with IgAN.

The present article takes a different approach to investigating the association of IgAN with the class II locus of the $\mathrm{MHC}$, in view of the considerable interest that has arisen in understanding patterns of linkage disequilibrium (LD) in the human genome to facilitate association studies involving complex diseases (Jeffreys et al. 2001). Singlenucleotide polymorphisms (SNPs) in particular are receiving attention as having potential influence on susceptibility to complex diseases, including IgAN (Takei et al. 2002). The ethnically homogeneous population of Japan (Usami et al. 2000) presents an opportunity to study genetic factors other than race/ethnicity that might contribute to the incidence of IgAN. We provide here an estimation of the extent of LD in the HLA class II locus, and we demonstrate linkage of IgAN to a gene in this region by means of a casecontrol association study involving a large number of Japanese patients and controls.

\section{Materials and methods}

\section{Materials}

Peripheral blood samples were obtained from 313 patients (176 women and 137 men, mean age of $44.2 \pm 14.3$ years) who were diagnosed with IgAN on the basis of clinical manifestations as well as renal-biopsy findings at one of several surgical centers in Japan (Division of Clinical Nephrology and Rheumatology, Niigata University Graduate School of Medical and Dental Sciences; Department of Medicine, Kidney Center, Tokyo Women's Medical University; Department of Urology, Iwate Medical University; Department of Urology, Iwate Prefectural Ofunato Hospital; and Department of Urology, Sanai Hospital). HenochSchönlein purpura and secondary IgAN such as hepatic glomerulosclerosis were excluded from the analysis. The mean value of serum creatinine at the time of renal biopsy was $1.07 \mathrm{mg} / \mathrm{dl}$, ranging from 0.3 to $2.5 \mathrm{mg} / \mathrm{dl}$. We analyzed DNA from 816 volunteers (492 women and 324 men, mean age of $54.4 \pm 14.5$ years) as controls. These healthy subjects without hematuria, proteinuria, and renal dysfunction were randomly selected from the Japanese population. Genomic DNA was prepared from each sample according to standard protocols. Informed consent was obtained from all participants.
Markers and genotyping

Information about each SNP in the HLA class II region chosen for this study was obtained from the Japanese SNP (JSNP) database (http://snp.ims.u-tokyo.ac.jp). We amplified multiple genomic fragments using $20 \mathrm{ng}$ of genomic DNA for each polymerase chain reaction (PCR), as described elsewhere (Ohnishi et al. 2000). Sequences of all primers are available at JSNP. Each PCR was performed in a $20-\mu \mathrm{l}$ solution containing $50 \mathrm{pmol}$ of each primer, 10 units of Ex-Taq DNA polymerase (TaKaRa Shuzo, Tokyo, Japan), and $0.55 \mu \mathrm{g}$ of TaqStart (CLONTECH Laboratories, Tokyo, Japan) in the GeneAmp PCR system 9700 (Applied Biosystems, Foster City, CA, USA). Initial denaturation was at $94^{\circ} \mathrm{C}$ for $2 \mathrm{~min}$, followed by 37 cycles of amplification at $94^{\circ} \mathrm{C}$ for $15 \mathrm{~s}$ and annealing at $60^{\circ} \mathrm{C}$ for $45 \mathrm{~s}$, with a final extension for $2 \mathrm{~min}$ at $72^{\circ} \mathrm{C}$. We genotyped each SNP by means of the Invader assay that combines a structure-specific cleavage enzyme with a universal fluorescent resonance energy transfer system (Mein et al. 2000).

\section{Typing of $H L A-D R B 1$ by DNA sequencing}

Using a technique of random sampling, we selected 82 of the IgAN patients and 253 of the controls. We typed these subjects for $H L A-D R B 1$ according to DNA sequence, using the HLA-DRB BigDye Terminator Sequencing-Based Typing Kit according to the manufacturer's instructions (Applied Biosystems).

\section{Statistical analysis}

Genotype distributions and allele frequencies of each selected SNP were compared, respectively, between cases and controls using the chi-square test. Significance was judged according to the guidelines of Lander and Kruglyak (1995). Odds ratios (ORs) and $95 \%$ confidence intervals (CIs) were calculated by Woolf's method. Hardy-Weinberg equilibrium was assessed by $\chi^{2}$ statistics (Nielsen et al. 1998). Frequencies of $H L A-D R B 1$ alleles were obtained by counting the total number of specific alleles. HLA-DRB1 allele frequencies in IgAN patients were assessed for significant deviation from those of the control group by means of the $\chi^{2}$ test, or by Fisher's exact test when criteria for the $\chi^{2}$ test could not be applied.

Analysis of linkage disequilibrium

We estimated maximum-likelihood haplotype frequencies for each pair of SNP markers from the genotypic data of 94 controls. We used these frequencies to estimate the level of LD between each pair of SNPs, using $\mathrm{D}^{\prime}$ value (Devlin and Risch 1995) for all pairs of markers with minor-allele frequencies of at least 0.10, except for SNPs not falling under the assumption of Hardy-Weinberg equilibrium. 


\section{Results}

LD mapping in the HLA class II region

The region analyzed in the present study covered genomic DNA between the DPB2 and TSBP genes on chromosome 6p21.3 (Fig. 1a). The LD patterns defined by 42 SNP markers are summarized in Fig. 1b. Because lower-frequency markers showed inconsistent LD patterns (Jeffreys et al. 2001), we selected markers with allelic frequencies of their minor alleles of greater than $10 \%$. The LD map constructed in this study revealed five extended blocks of high disequilibrium that broke down at the BTNL-2, DQA2, LMP2, and $D O A$ loci (Fig. 1b).

Case-control study in each domain

To investigate a possible association between $\operatorname{IgAN}$ and SNPs in each block, we genotyped 313 patients with IgAN and 816 controls at the five loci listed in Table 1 . The genotype distributions we observed in controls did not differ from the expected frequency under the assumption of Hardy-Weinberg equilibrium (data not shown). A significant association to IgAN was observed at the $D R A$ locus, but no association was found at the remaining four loci (Table 1).

In view of the strong association found at the $D R A$ locus, we genotyped six SNPs present in the $H L A-D R A$ gene (Fig. $2)$. The most significant difference in genotype distribution between patients with IgAN and controls was observed at the DRA SNP-5 locus (Table 2). Homozygosity for major alleles was significantly more common in $\operatorname{IgAN}$ patients than in controls $\left(\chi^{2}=22.87, P=0.000001\right)$. The OR for patients with IgAN versus controls was 1.91 (95\% CI 1.462.49) for homozygotes of the DRA SNP-5 major allele versus others. One of the three SNPs for which we found positive associations would alter an amino acid sequence: DRA SNP-6, which showed complete LD to DRA SNP-2, would substitute valine for leucine at codon 222 of the $H L A-D R A$ gene $\left(\chi^{2}=19.96, P=0.00004\right)$. The OR for patients with IgAN versus controls was 1.77 (95\% CI 1.362.31) for homozygotes of the DRA SNP-6 major allele versus others. In contrast, no significant differences were observed for DRA SNP-3 or DRA SNP-4.

Distribution of $H L A-D R B 1$ alleles

Because the HLA-DRB region lies in close vicinity to $D R A$, we also examined the relationship between the DRB region and SNPs for susceptibility to $\operatorname{IgAN}$. Because $D R B 1$ is highly polymorphic, we determined the genotypes of 82 IgAN patients and 253 controls by direct DNA sequencing. As shown in Table 3, the frequency of DRB1*04 tends to be higher in patients than in controls $(P=0.034)$, but the association of the $D R B 1$ gene to IgAN was less significant than that of the $D R A$ gene.

\section{Discussion}

We have examined the extent and strength of LD within the class II locus of MHC in a Japanese population sample.

Table 1. Genotype frequencies and association tests of SNPs in the class II region (313 cases of IgAN vs 816 controls)

\begin{tabular}{|c|c|c|c|c|c|}
\hline & $D O A$ & $D M B$ & $D Q B 2$ & $D R A$ & $T S B P$ \\
\hline \multicolumn{6}{|l|}{ SNP information } \\
\hline Contig number & NT_007592.8 & NT_007592.8 & NT_007592.8 & NT_007592.8 & NT_007592.8 \\
\hline Location & 15399187 & 15328808 & $151 \overline{5} 1100$ & 14860033 & $147 \overline{8} 3966$ \\
\hline Genetic variation & $\mathrm{T}>\mathrm{C}$ & $\mathrm{C}>\mathrm{A}$ & $\mathrm{A}>\mathrm{G}$ & $\mathrm{C}>\mathrm{T}$ & $\mathrm{C}>\mathrm{T}$ \\
\hline \multicolumn{6}{|l|}{$\operatorname{IgAN}$} \\
\hline Major allele & 0.61 & 0.45 & 0.68 & 0.66 & 0.65 \\
\hline Minor allele & 0.39 & 0.55 & 0.32 & 0.34 & 0.35 \\
\hline Total & 1.00 & 1.00 & 1.00 & 1.00 & 1.00 \\
\hline Major homozygous & 0.36 & 0.23 & 0.49 & 0.46 & 0.44 \\
\hline Heterozygous & 0.49 & 0.45 & 0.37 & 0.39 & 0.42 \\
\hline Minor homozygous & 0.15 & 0.32 & 0.14 & 0.15 & 0.14 \\
\hline Total & 1.00 & 1.00 & 1.00 & 1.00 & 1.00 \\
\hline \multicolumn{6}{|l|}{ Control } \\
\hline Major allele & 0.64 & 0.51 & 0.68 & 0.55 & 0.61 \\
\hline Minor allele & 0.36 & 0.49 & 0.32 & 0.45 & 0.39 \\
\hline Total & 1.00 & 1.00 & 1.00 & 1.00 & 1.00 \\
\hline Major homozygous & 0.41 & 0.26 & 0.46 & 0.31 & 0.37 \\
\hline Heterozygous & 0.45 & 0.50 & 0.44 & 0.48 & 0.48 \\
\hline Minor homozygous & 0.14 & 0.24 & 0.10 & 0.21 & 0.15 \\
\hline Total & 1.00 & 1.00 & 1.00 & 1.00 & 1.00 \\
\hline \multicolumn{6}{|l|}{$\chi^{2}[P]$} \\
\hline Genotype frequency $(2 \times 3$ table $)$ & $2.32[0.3]$ & $7.37[0.02]$ & $6.52[0.03]$ & $23.04[0.000009]$ & $5.05[0.08]$ \\
\hline Allele frequency (major vs minor) & $1.77[0.1]$ & $5.46[0.01]$ & $0.06[0.8]$ & $19.82[0.000008]$ & $3.64[0.05]$ \\
\hline Major homozygous vs others & $2.32[0.1]$ & $1.13[0.2]$ & $0.79[0.3]$ & $22.87[0.000001]$ & $5.03[0.02]$ \\
\hline Minor homozygous vs others & $0.23[0.6]$ & $7.35[0.006]$ & $3.86[0.04]$ & $4.37[0.03]$ & $0.36[0.5]$ \\
\hline
\end{tabular}

SNP, Single-nucleotide polymorphism 


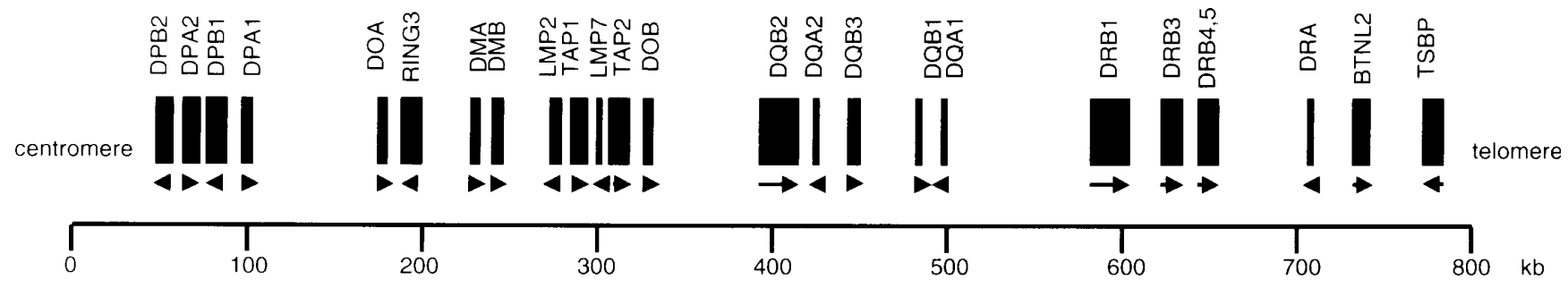

a
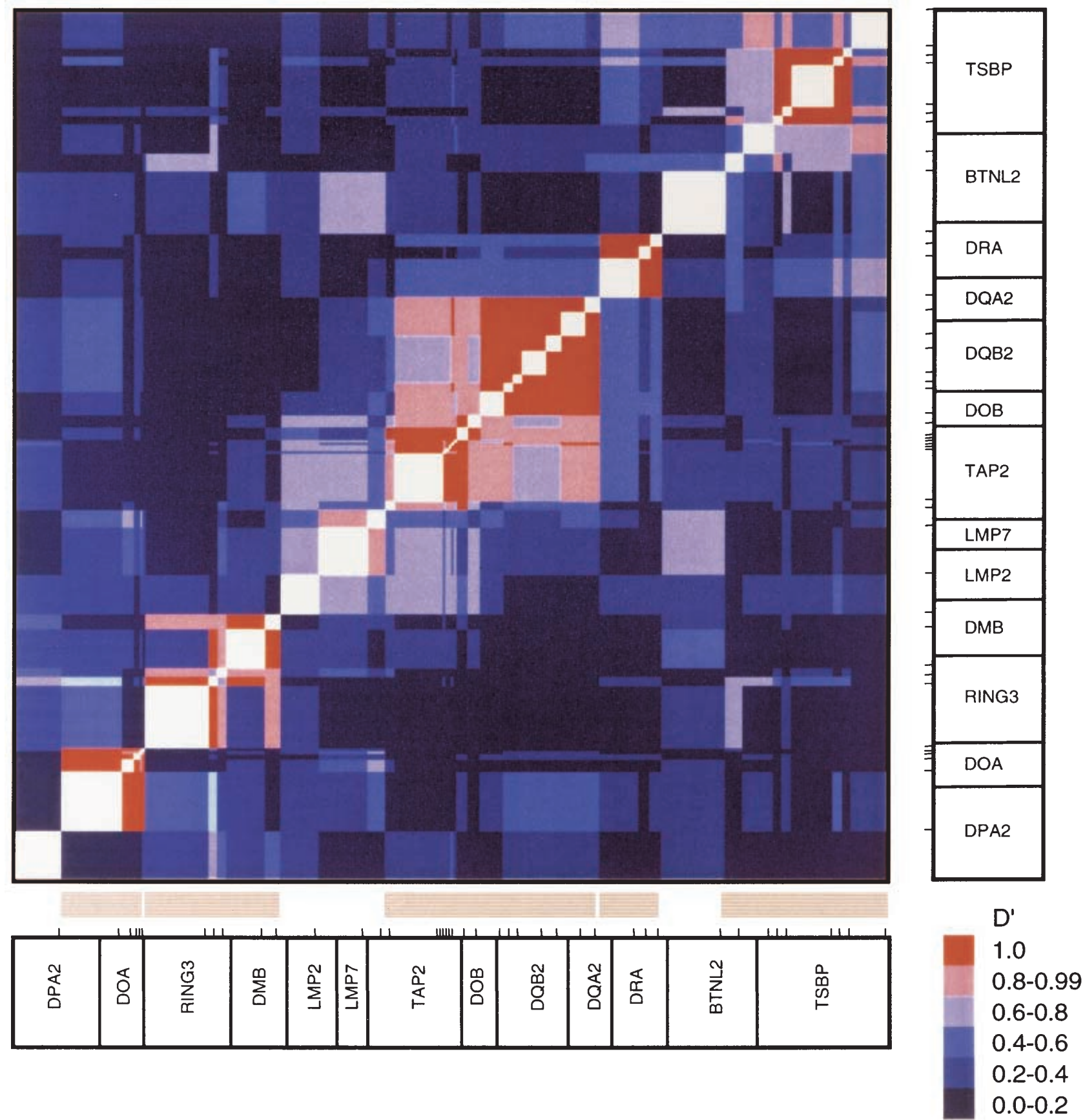

Fig. 1. a The genomic region extending from $D P B 2$ to $T S B P$ on human chromosome 6p21.3. b Distribution of linkage disequilibrium (LD) in the class II region, adjusted for physical distance. Single- nucleotide polymorphism (SNP) sites are indicated by tick marks at their locations in the respective genes. Domains showing strong LD are indicated below the chart in light crimson 


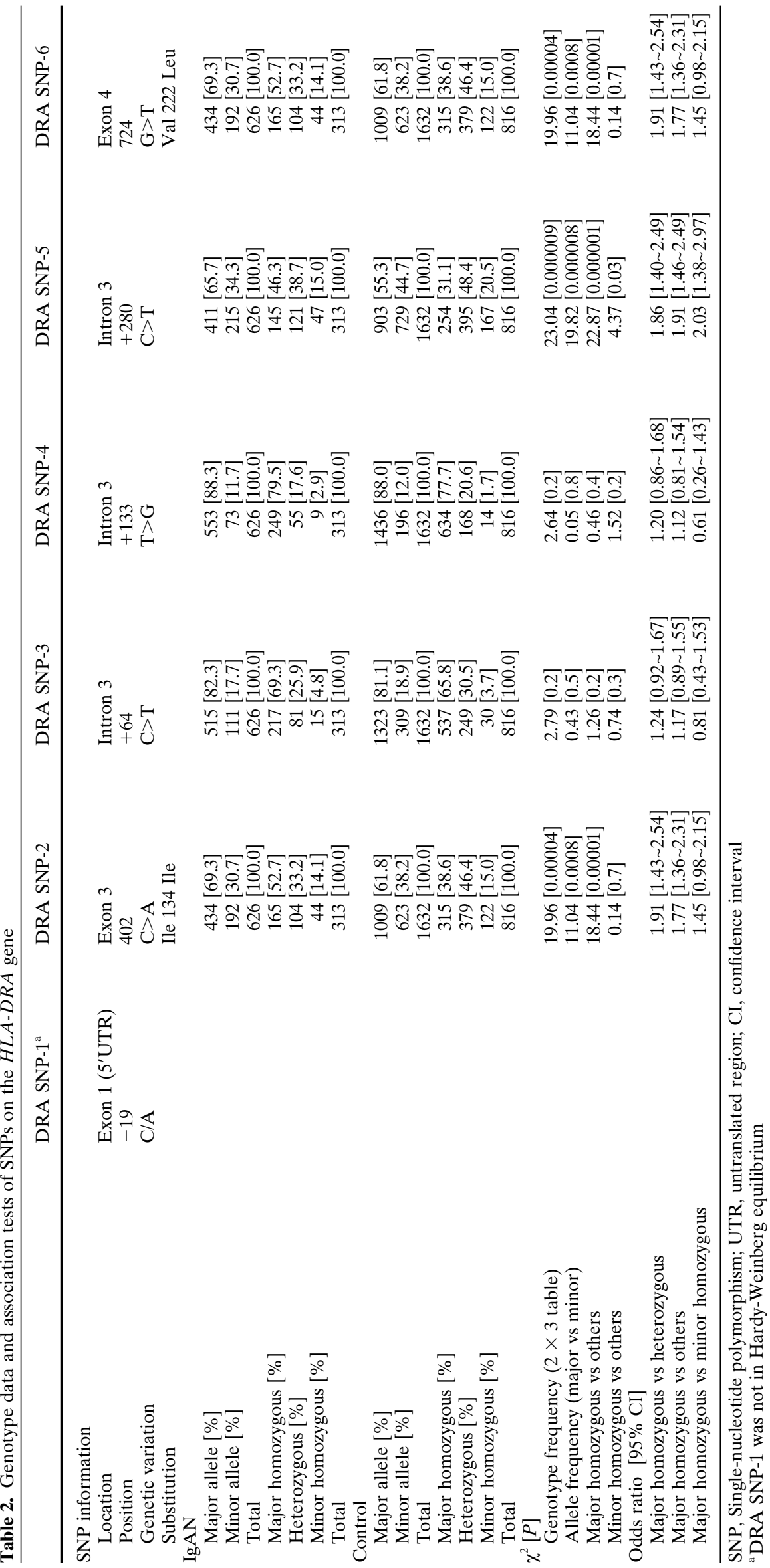




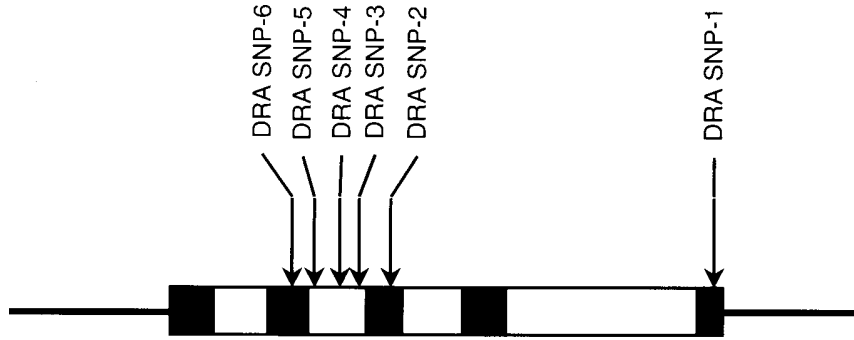

$H L A-D R A$

Fig. 2. Location of SNPs in the $H L A-D R A$ gene

Table 3. Gene frequencies (\%) of $H L A-D R B 1$ alleles in patients with IgAN and controls

\begin{tabular}{lll}
\hline & \multicolumn{2}{l}{ Group } \\
\cline { 2 - 3 } DRB1 allele & IgAN $(n=82)$ & $\begin{array}{l}\text { Controls }(n=253) \\
\text { 164 alleles }\end{array}$ \\
\hline$* 1$ & 3.1 & 5.7 \\
$* 15$ & 15.2 & 18.4 \\
$* 04$ & $26.2^{\dagger}$ & 18.4 \\
$* 11$ & 2.4 & 3.4 \\
$* 12$ & 3.1 & 4.7 \\
$* 13$ & 7.3 & 8.5 \\
$* 14$ & 13.4 & 11.5 \\
$* 07$ & 0.6 & 0.4 \\
$* 08$ & 11.0 & 12.1 \\
$* 09$ & 17.1 & 14.8 \\
$* 10$ & 0.6 & 1.4 \\
\hline
\end{tabular}

${ }^{\dagger} P=0.034$

Many factors influence the recombination rate and extent of $\mathrm{LD}$, but a remarkable similarity of LD patterns in the MHC region has been observed in populations whose genetic and demographic histories are vastly different (Zavattari et al. 2000). The distribution of crossover events in the class II region tends to cluster in three hot spots, namely, between $D Q B 1$ and $D Q B 3$, between RING3 and $D O A$, and in a region within the TAP2 gene (Zavattari et al. 2000). The LD blocks in our map were separated by corresponding intervals, defined as $D R A$ and $D Q A 2, R I N G 3$ and $D O A$, and $T A P 2$ and $D M B$, indicating consistency with other studies (Jeffreys et al. 2001).

LD is a situation in which two closely located polymorphisms show association with each other. LD enables us to use an allele of one SNP to predict an allele of another (nearby) polymorphism. Any potential instance of LD between an SNP and a disease-causing, functional polymorphism (which might also be an SNP) is the basis for whole-genome association studies designed to detect genes involved in complex diseases (Remm and Metspalu 2002).

We demonstrate that the frequencies of DRB $1 * 04$ was increased in patients with $\operatorname{IgAN}$, consistent with other previous reports that HLA-DR4 was associated with IgAN in a Japanese population $(P<0.04)$, although the reported $P$ value was not significantly small (Hiki et al. 1982; Kashiwabara et al. 1982). Moreover, the apparent association between $H L A-D R A$ alleles and IgAN has not been clarified in the Japanese or any other ethnic group; we have demonstrated here for the first time a significant association of three SNPs in the HLA-DRA gene with IgAN. However, because the $D Q A 1$ and $D Q B 1$ loci, which lie within the same LD domain, are highly polymorphic and remain untyped, we cannot exclude the possibility of an association of either or both of these genes with susceptibility to IgAN.

The class II region of the MHC contains a number of interesting candidates for susceptibility to a variety of diseases because of their polymorphic features and the antigenicity of their products. Strong associations exist between products of the polymorphic HLA-DR alleles and certain autoimmune diseases because HLA-DR molecules are of great importance in the selection and activation of CD4positive $\mathrm{T}$ cells that regulate immune responses against protein antigens (Vyse and Todd 1996). However, the pathophysiology of these autoimmune disorders is not completely understood.

Class II molecules are composed of an alpha chain that is noncovalently associated with a beta chain encoded by the A and B gene loci, respectively, in MHC, and are expressed primarily on antigen-processing cells such as dendritic cells, B lymphocytes, and macrophages. The DR molecule consists a single alpha chain encoded by the $D R A$ gene and four species of beta chain encoded by the $D R B 1$, $D R B 3, D R B 4$, and DRB5 genes. For Class II, both the A and $B$ genes contribute to variable $\alpha-1$ and $\beta-1$ domains that form a peptide-binding cleft (Williams 2001). The SNPs for which we found positive association with $\operatorname{IgAN}$ are not located in this variable $\alpha-1$ domain. However, because the amino-acid substitution caused by the DRA SNP-6 occurs in the intracellular domain of the DRA molecule, it may affect the structures of peptides bound to HLA class II antigens.

The fundamental role of class II molecules is to bind to self and nonself peptides and transport them to the plasma membrane of cells for recognition by the T-cell antigen receptor. DRA SNP-6 may bring about individual differences in immune responses by influencing signals for alternative pathways involving internalization of HLA-DR molecules (Stern et al. 1994; Pinet et al. 1995). It is well known that, in autoimmune diseases, the activation of autoreactive CD4-positive $\mathrm{T}$ cells, which are inactivated under normal conditions, is considered to be a crucial step in the development of disease. Because the IgA antibody response is T-cell dependent, the MHC class II products encoded by DR genes might play a crucial role in the presentation of processed antigen to specific $\mathrm{T}$ cells (Hsu et al. 2000). However, the exact mechanism by which the DRA molecule contributes to the development of IgAN remains to be determined.

Acknowledgments We gratefully acknowledge assistance from Kyoko Kobayashi, Susumu Saito, Akihiro Sekine, and technicians at the SNP Research Center, The Institute of Physical and Chemical Research (RIKEN). This work was supported in part by a "Research for the 
Future" Program Grant of The Japan Society for the Promotion of Science to Y.N.

\section{References}

Devlin B, Risch N (1995) A comparison of linkage disequilibrium measures for fine-scale mapping. Genomics 29:311-322

Floege J, Feehally J (2000) IgA nephropathy: recent developments. J Am Soc Nephrol 11:2395-2403

Galla JH (1995) IgA nephropathy. Kidney Int 47:377-387

Galla JH (2001) Molecular genetics in IgA nephropathy. Nephron 88:107-112

Hiki Y, Kobayashi Y, Tateno S, Sada M, Kashiwagi N (1982) Strong association of HLA-DR4 with benign IgA nephropathy. Nephron 32:222-226

Hsu SIH, Ramirez SB, Winn MP, Bonventre JV, Owen WF (2000) Evidence for genetic factors in the development and progression of IgA nephropathy. Kidney Int 57:1818-1835

Jeffreys AJ, Kauppi L, Neumann R (2001) Intensely punctate meiotic recombination in the class II region of the major histocompatibility complex. Nat Genet 29:217-222

Julian BA, Quiggins PA, Thompson JS, Woodford SY, Gleason K, Wyatt RJ (1985) Familial IgA nephropathy. Evidence of an inherited mechanism of disease. N Engl J Med 312:202-208

Kashiwabara H, Shishido H, Tomura S, Tuchida H, Miyajima T (1982) Strong association between IgA nephropathy and HLA-DR4 antigen. Kidney Int 22:377-382

Lander E, Kruglyak L (1995) Genetic dissection of complex traits: guidelines for interpreting and reporting linkage results. Nat Genet 11:241-247

Mein CA, Barratt BJ, Dunn MG, Siegmund T, Smith AN, Esposito L, Nutland S, Stevens HE, Wilson AJ, Phillips MS, Jarvis N, Law S, de Arruda M, Todd JA (2000) Evaluation of single nucleotide polymorphism typing with invader on PCR amplicons and its automation. Genome Res 10:330-343

Moore R (1993) MHC gene polymorphism in primary IgA nephropathy. Kidney Int 43(Suppl 39):S9-S12

Nielsen DM, Ehm MG, Weir BS (1998) Detecting marker-disease association by testing for Hardy-Weinberg disequilibrium at a marker locus. Am J Hum Genet 63:1531-1540

Ohnishi Y, Tanaka T, Yamada R, Suematsu K, Minami M, Fujii K, Hoki N, Kodama K, Nagata S, Hayashi T, Kinoshita N, Sato H, Sato
H, Kuzuya T, Takeda H, Hori M, Nakamura Y (2000) Identification of 187 single nucleotide polymorphisms (SNPs) among 41 candidate genes for ischemic heart disease in the Japanese population. Hum Genet 106:288-292

Pinet V, Vergelli M, Martin R, Bakke O, Long EO (1995) Antigen presentation mediated by recycling of surface HLA-DR molecules. Nature 375:603-606

Remm M, Metspalu A (2002) High-density genotyping and linkage disequilibrium in the human genome using chromosome 22 as a model. Curr Opin Chem Biol 6:24-30

Sabatier JC, Genin C, Assenat H, Colon S, Ducret F, Berthoux FC (1979) Mesangial IgA glomerulonephritis in HLA-identical brothers. Clin Nephrol 11:35-38

Schena FP (1995) Immunogenetic aspects of primary IgA nephropathy. Kidney Int 48:1998-2013

Scolari F, Amoroso A, Savoldi S, Mazzola G, Prati E, Valzorio B, Viola BF, Nicola B, Movilli E, Sandrini M, Campanini M, Maiorca R (1999) Familial clustering of $\operatorname{Ig} A$ nephropathy: further evidence in an Italian population. Am J Kidney Dis 33:857-865

Stern LJ, Brown JH, Jardetzky TS, Gorga JC, Urban RG, Strominger JL, Wiley DC (1994) Crystal structure of the human class II MHC protein HLA-DR1 complexed with an influenza virus peptide. Nature 368:215-221

Takei T, Iida A, Nitta K, Tanaka T, Ohnishi Y, Yamada R, Maeda S, Tsunoda T, Takeoka S, Ito K, Honda K, Uchida K, Tsuchiya K, Suzuki Y, Fujioka T, Ujiie T, Nagane Y, Miyano S, Narita I, Gejyo F, Nihei H, Nakamura Y (2002) Association between singlenucleotide polymorphisms in selectin genes and immunoglobulin A nephropathy. Am J Hum Genet 70:781-786

Tolkoff-Rubin NE, Cosimi AB, Fuller T, Rubin RH, Colvin RB (1978) IgA nephropathy in HLA-identical siblings. Transplantation 26:430433

Usami T, Koyama K, Takeuchi O, Morozumi K, Kimura G (2000) Regional variations in the incidence of end-stage renal failure in Japan. JAMA 284:2622-2624

Vyse TJ, Todd JA (1996) Genetic analysis of autoimmune disease. Cell 85:311-318

Williams TM (2001) Human leukocyte antigen gene polymorphism and the histocompatibility laboratory. J Mol Diagn 3:98-104

Zavattari P, Deidda E, Whalen M, Lampis R, Mulargia A, Loddo M, Eaves I, Mastio G, Todd JA, Cucca F (2000) Major factors influencing linkage disequilibrium by analysis of different chromosome regions in distinct populations: demography, chromosome recombination frequency and selection. Hum Mol Genet 9:29472957 